\title{
Fundamental Aerodynamics of a New Volleyball
}

Short running head title: Aerodynamics of a New Volleyball

Takeshi Asai $^{1)}$, Shinichiro Ito $^{2)}$, Kazuya Seo ${ }^{3)}$, Akihiro Hitotsubashi ${ }^{4)}$

1) Takeshi Asai, Ph. D. (Correspondent author)

Inst. of Health and Sports Sci., B207

Comprehensive Human Sciences

University of Tsukuba

Tsukuba-city, 305-8574, Japan.

Tel. \& Fax: (029) 853-2711

Email: asai@taiiku.tsukuba.ac.jp

2) Shinichiro Ito, Ph. D.

Department of Fluid Mech., Kogakuin University,

1-24-2 Nishi-Shinjuku, Shinjuku-ku,

Tokyo, 163-8677, Japan.

Tel. (03) 3340-2648, FAX: (03)3340-0108

Email: ito@cc.kogakuin.ac.jp

3) Kazuya Seo, Ph. D.

Faculty of Education, Yamagata Univ.,

1-4-12, Koshirakawa, Yamagata-city,

Yamagata, 990-8560, Japan.

Tel. (023) 628-4350, FAX: (023)628-4454

Email: seo@e.yamagata-u.ac.jp 
4) Akihiro Hitotsubashi,

Molten corp., Nakahiromachi 1-5-8,

Nishi-ku, Hiroshima, 733-0012, Japan.

Tel. (082) 232-5627, FAX: (082)232-2931

Email: hitotsubashi@molten.co.jp

Abstract; 180 words

Main text; 1768 words

8 figures (16 panels) 


\begin{abstract}
The purpose of this study is to analyze the basic aerodynamic characteristics of conventional volleyballs and new volleyballs by using a wind tunnel. Furthermore, we used an impact-type ball ejection device to measure the values of the coordinates of the point at which the ball lands near the critical Reynolds regime $(14 \mathrm{~m} / \mathrm{s})$. It was founded that the critical Reynolds number for conventional volleyball (molten MTV5SLIT) was approximately 270,000 and that for the new volleyball (honeycomb type: molten V5M5000) was approximately 220,000. The Reynolds number of the new volleyball was slightly lower than the conventional volleyball. New balls (honeycomb type) showed a drag coefficient of approximately 0.17 when supercritical, a rather large figure when component to the 0.1 coefficient of conventional balls. One reason for this may be that the honeycombed protrusion design increases the roughness of the ball. It is considered that the honeycombed protrusion design of new balls increases the roughness of the ball surface, and the increase in roughness within the parameters of our tests implies a trend towards reduced aerodynamic instability close to critical Reynolds numbers.
\end{abstract}

\title{
Keywords
}

Aerodynamics; Ball; Volleyball; Drag; Instability 


\section{Introduction}

Many studies have been conducted on the aerodynamics of sports balls including golf (Bearman and Harvey, 1976; Smits and Ogg, 2004), cricket (Mehta et al., 1983), tennis (Haake et al., 2000; Mehta and Pallis, 2001), baseball (Watts and Ferrer, 1987), rugby balls (Seo et al., 2004) and soccer balls (Asai et al., 2007). Mehta (1985) carried out the first proper review of sports ball aerodynamics by covering in depth the sports of cricket, golf and baseball. Mehta (1985) discussed that the drag on a ball was dominated by the size and deflection of the wake. However, there have been few such studies on volley balls (Wei et al., 1988; Cairns, 2004). Specifications for volleyballs are determined by the Fédération Internationale de Volleyball (FIVB). New volleyballs that meet these specifications are being routinely manufactured and are used in competitions and other places where the sport is played. However, the aerodynamic characteristics of these newly manufactured volleyballs have not been clarified.

The purpose of this study is to analyze the basic aerodynamic characteristics of conventional volleyballs and new volleyballs by using a wind tunnel. Furthermore, we used an impact-type ball ejection device to measure the values of the coordinates of the point at which the ball lands near the critical Reynolds regime $(R e=220,000)$. Thus, we were able to examine the aerodynamic instability at that speed range.

\section{Methods}

\subsection{Wind tunnel test}


Three full-size FIVB official volley balls were used to determine the aerodynamic forces acting on each ball in a low-speed wind tunnel with a $0.7 \mathrm{~m} \times 0.7 \mathrm{~m}$ rectangular cross-section area (turbulence level $\leq 1 \%$ ). The tested volleyballs were one conventional type volleyball (molten MTV5SLIT) and two new type volleyballs (molten V5M5000 and mikasa MVA200) (Figure 1). The surface of the conventional volleyball is relatively flat (Figure 1a), the molten V5M5000 has a small honeycombed protrusion design (Figure 1b), and the mikasa MVA200 has small dimples similar to those on a golf ball (Figure 1c).

The volleyball was used together with a stainless steel rod as shown in Figure 2. Data were acquired by using a 3-component strut-type balance (LMC-3531-50NS; Nissho Electric Works) over a period of $4.096 \mathrm{~s}$ and recorded on a personal computer with the aid of an A/D converter board. The sampling rate was 1,000 per second. Aerodynamic force data were taken for various wind speeds, $U$, between 6 and $30 \mathrm{~m} / \mathrm{s}$. The following parameters were calculated from experimental data collected over a range of conditions: the force acting in the direction opposite to the direction of the wind, known as drag $(D)$ and the force acting sideways on the basis of a frontal view $(L)$. The aerodynamic forces measured in the experiment were converted to the drag coefficient $(C d)$ and the lift coefficient $(C l)$, as shown in equations 1 and 2 .

$$
C d=\frac{D}{\frac{1}{2} \rho U^{2} A} \quad(1) \quad C l=\frac{L}{\frac{1}{2} \rho U^{2} A}
$$


In the above equations, $\rho$ is the density of air $\left(1.2 \mathrm{~kg} / \mathrm{m}^{3}\right), U$ is the flow velocity $(\mathrm{m} / \mathrm{s})$, and $A$ is the projected area $\left(\mathrm{m}^{2}\right)$ of the volleyball.

\subsection{The aerodynamic instability test using an impact-type ball ejection device}

We examined the aerodynamic instability of the volleyball near the critical Reynolds regime; we used an impact-type ball ejection device (Figure 3) to measure the coordinate values at the landing point of the ball in flight. We utilized a low-profile flat-impact unit to propel the volleyball, and we measured the variation in the landing point of a ball traveling with almost no rotation. This test was performed indoors to minimize the effects of natural air currents; we used 3 types of volleyballs (conventional volleyball: molten MTV5SLIT, new volleyball: molten V5M5000, and new volleyball: mikasa MVA200) for this test.

We used 3 types of orientations (Face A, Face B and Face C) and 10 measurements were made for each orientation (Figure 4a). The distance between the impact-style ball ejection device and the point of landing is represented by L, while the horizontal variation is represented by C (Figure 4b). By default, the rotation of the ball is almost nil and the flight characteristics appear to mimic those of a "floater serve.” The initial velocity of the ball in this experiment is approximately $15 \mathrm{~m} / \mathrm{s}$ (Re $=217,000)$. As compared to the deviations in serves measured from human players, these results appear relatively reliable. Ejecting a ball using a horizontal frontal impact on the panel as in Face A produced extreme horizontal and vertical variations in the landing point, while direct impacts on the valve in Face $\mathrm{C}$ and ejecting it vertically resulted in a compact landing pattern. The following factors may have an influence on the landing point of the ball: the slipstream caused by the separation of the boundary layer from the connecting area of the panel, the effect on the boundary 
layer developed by the valve, the small amount of initial rotation of the ball, eccentricities in the centre of gravity, and vibration in the ball receiver.

\section{Results and Discussion}

\subsection{Drag coefficient and Lift coefficient}

On the basis of these experiments, we found that the critical Reynolds number for conventional volleyball (molten MTV5SLIT) was approximately 270,000, that for the new volleyball (honeycomb type: molten V5M5000) was approximately 220,000 and for the new volleyball (dimple type: Mikasa MVA200) was approximately 310,000 The Reynolds number of the new honeycomb type volleyball (molten V5M5000) was slightly lower than the conventional volleyball (Figure 5). Furthermore, the critical Reynolds number for new dimple type volleyballs of a different type (Mikasa MVA200) is approximately 310,000, and in our testing we demonstrated a smaller figure. New balls (honeycomb type) showed a drag coefficient of approximately 0.17 when supercritical, a rather large figure when component to the 0.1 coefficient of conventional balls. One reason for this may be that the honeycombed protrusion design increases the roughness of the ball. The lift coefficient for both types of volleyballs showed a large degree of instability at certain speed ranges, but we did not observe any consistent trend (Figure 6).

\subsection{Comparing the deviation between the coordinate values at the landing point} The impact (landing) points for a new ball (honeycomb type) ejected from an impact type ball ejection device with an initial speed of $15 \mathrm{~m} / \mathrm{s}$ exhibit in the horizontal range of approximately $\pm 2 \mathrm{~m}$ and the lateral range of approximately $4 \mathrm{~m}$ (Figure 7a). 
Moreover, A, B, and C in the figure show the orientation of the ball panels when the ball is ejected; a trend for the impact point to vary with panel orientation. This suggests that the surface panel orientation when the ball is ejected alters the flow around the ball when it is in flight and affects the flight trajectory. Comparing the impact points for different ball types demonstrates that honeycomb-type new balls (honeycomb type) exhibit less dispersion than conventional balls and dimple-type new balls (Figure 7a, 7b and 7c). When comparing standard deviations for the degree of dispersion in impact points at this time, flight direction for Type A (honeycomb) new balls shows a standard deviation of 0.91 , and 0.53 for horizontal dispersion, while Type B conventional balls show a standard deviation of 0.99 for flight direction, and 0.54 for horizontal dispersion. Type C (dimple) new balls demonstrated a standard deviation of 1.14 for flight direction and 0.71 for horizontal dispersion. Thus, honeycomb type new balls show smaller standard deviations than other balls (Figure 8). These facts suggest that the honeycombed protrusion design of new balls increases the roughness of the ball surface, and the increase in roughness within the parameters of our tests implies a trend towards reduced aerodynamic instability close to critical Reynolds numbers.

\section{Conclusions}

The purpose of this study is to analyze the basic aerodynamic characteristics of conventional volleyballs and new volleyballs by using a wind tunnel. Furthermore, we used an impact-type ball ejection device to measure the values of the coordinates of the point at which the ball lands near the critical Reynolds regime $(14 \mathrm{~m} / \mathrm{s})$. Thus, we were able to examine the aerodynamic instability at that speed range. The results may 
be summarized as follows. It was founded that the critical Reynolds number for conventional volleyball (molten MTV5SLIT) was approximately 270,000 and that for the new volleyball (honeycomb type: molten V5M5000) was approximately 220,000. The Reynolds number of the new volleyball was slightly lower than the conventional volleyball. New balls (honeycomb type) showed a drag coefficient of approximately 0.17 when supercritical, a rather large figure when component to the 0.1 coefficient of conventional balls. One reason for this may be that the honeycombed protrusion design increases the roughness of the ball. It is suggested that the surface panel orientation when the ball is ejected alters the flow around the ball when it is in flight and affects the flight trajectory. It is considered that the honeycombed protrusion design of new balls increases the roughness of the ball surface, and the increase in roughness within the parameters of our tests implies a trend towards reduced aerodynamic instability close to critical Reynolds numbers.

\section{References}

Asai, T., Seo, K., Kobayashi, O. and Sakashita, R. (2007) Fundamental aerodynamics of the soccer ball, Sports Engineering, ISEA, 10(2), 101-109.

Bearman, P. W. and Harvey, J. K. (1976) Golf ball aerodynamics. Aeronautical Quarterly, 27, 112-122.

Cairns, T.W. (2004) Modeling the Lift and Drag Forces on a Volleyball. The Engineering of Sport 5 (Hubbard M., Mehta, R.D. and Pallis, J.M., eds.) Proceedings of the 5th International Sports Engineering, Association Conference, Davis, California, USA. Vol 1, 97-103.

Haake, S. J., Chadwick, S. G., Dignall, R. J., Goodwill, S. R, and Rose, P. (2000) Engineering tennis - slowing the game down. Sports Engineering, 3 (2), 131-143. 
Mehta, R.D., Bentley, K., Proudlove, M. and Varty, P. (1983) Factors affecting cricket ball swing. Nature, 303, 787-788.

Mehta R. D. (1985) Aerodynamics of Sports Balls, Annual Review of Fluid Mechanics, 17, 151-189.

Mehta, R. and Pallis, J. (2001) The aerodynamics of a tennis ball, Sports Engineering 4(4), 177-189.

Seo K., Kobayashi O. \& Murakami M. (2004) Regular and irregular motion of a rugby football during flight. IN: The Engineering of Sport 5, Vol. 1, (eds. M. Hubbard, R.D. Mehta \& J.M. Pallis), pp. 567-573. The International Sports Engineering Association, Sheffield.

Smits, A.J. and Ogg, S. (2004) Golf ball aerodynamics. The Engineering of Sport 5 (Eds. M. Hubbard, R.D. Mehta and J.M. Pallis). Pub. The International Sports Engineering Association, Sheffield, UK, 3-12.

Watts, R.G. and Ferrer, R. (1987) The Lateral Force on a Spinning Sphere: Aerodynamics of a Curvebal. Am. J. Phys., 55, 40-44.

Wei, Q., Lin, R. \& Liu, Z. (1988) Vortex-induced dynamics loads on a non-spinning volleyball. Fluid Dynamics Research, 3, 231-237. 


\section{List of Figures}

Figure 1. Ball panels (a, b, c) and shape of surfaces (d, e, f) on three type volleyballs for this experiment (a: molten MTV5SLIT, b: molten V5M5000, c: mikasa MVA200).

Figure 2. Set up of wind tunnel test (a) and the impact-type ball ejection device (b).

Figure 3. Set up of the impact-type ball ejection device (b).

Figure 4. Orientation of ball panels on volley ball (a) and Set up for the aerodynamic instability test (b).

Figure 5. Drag coefficient versus Reynolds number for conventional type (Old) and new type (New) volleyballs.

Figure 6. Lift coefficient versus Reynolds number for conventional type (Old) and new type (New) volleyballs.

Figure 7. Impact (landing) points for honeycomb type ball (a), conventional type ball (b) and dimple type ball (c) ejected from the impact type ball ejection device (A, B, and C in the figure show the orientation of the ball panels).

Figure 8. Standard deviations of longitudinal and transversal displacement for honeycomb type ball (Type-A), conventional type ball (Type-B) and dimple type ball (Type-C). 


\section{Figures}
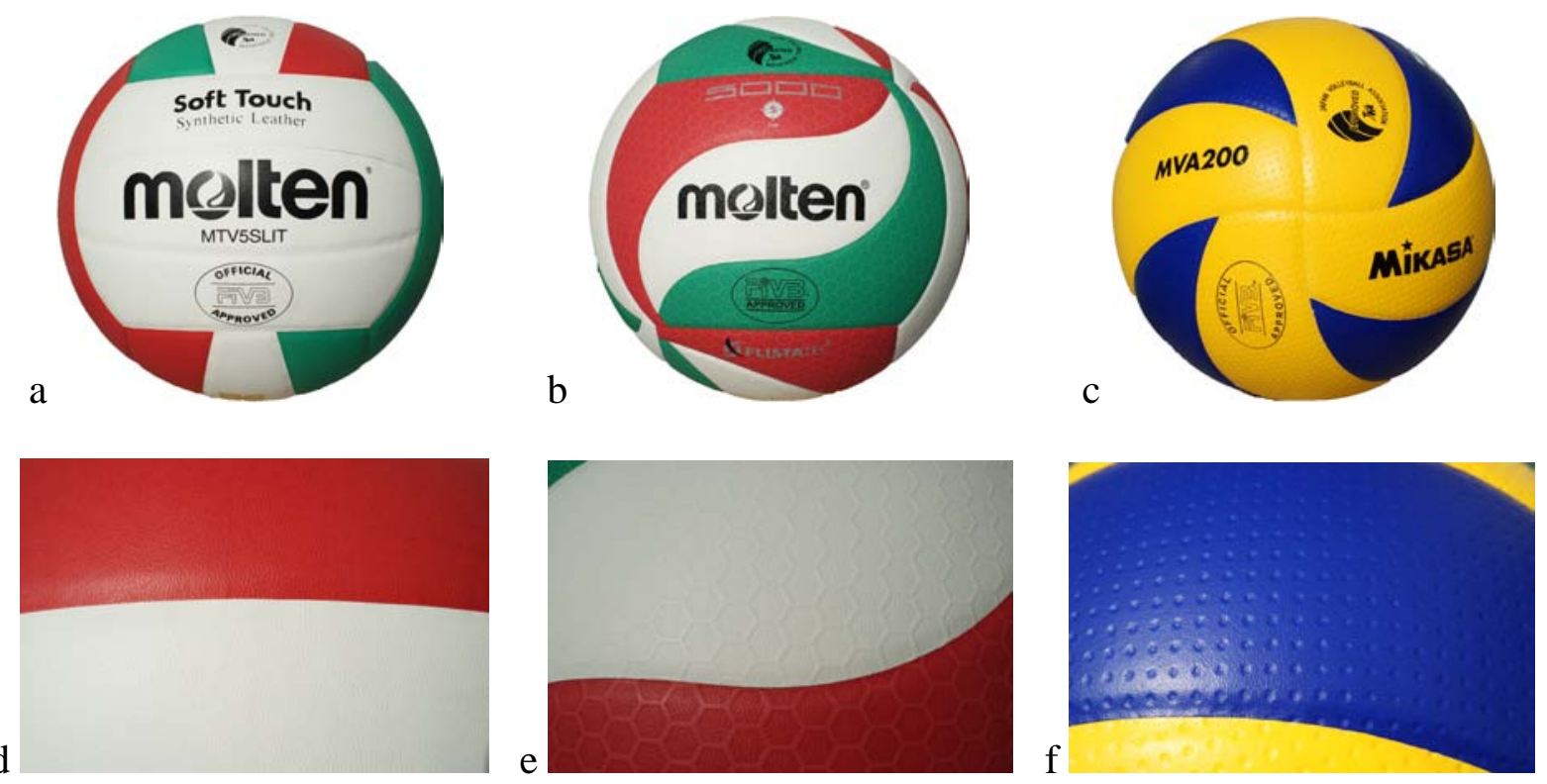

Figure 1. Ball panels (a, b, c) and shape of surfaces (d, e, f) on three type volleyballs for this experiment (a: molten MTV5SLIT, b: molten V5M5000, c: mikasa MVA200). 


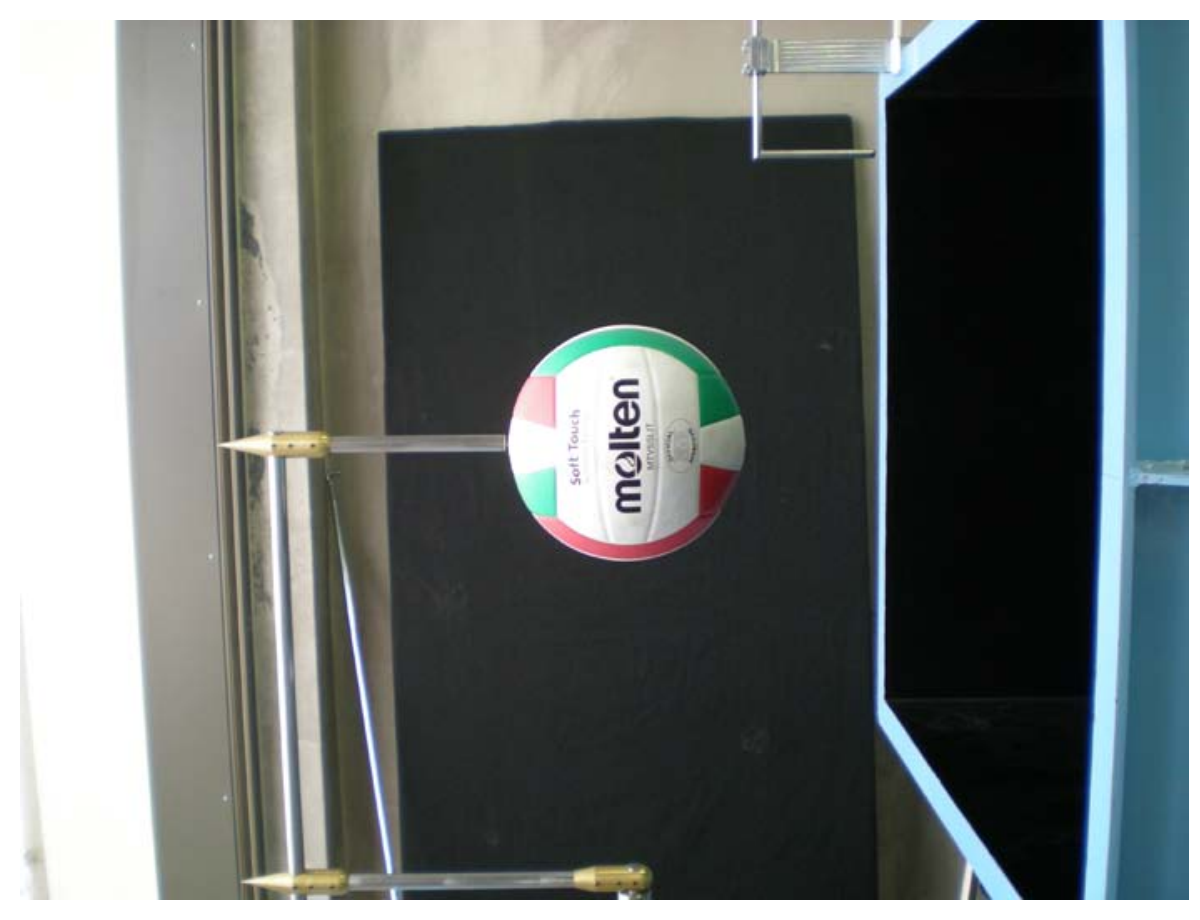

Figure 2. Set up of wind tunnel test (a) and the impact-type ball ejection device (b). 


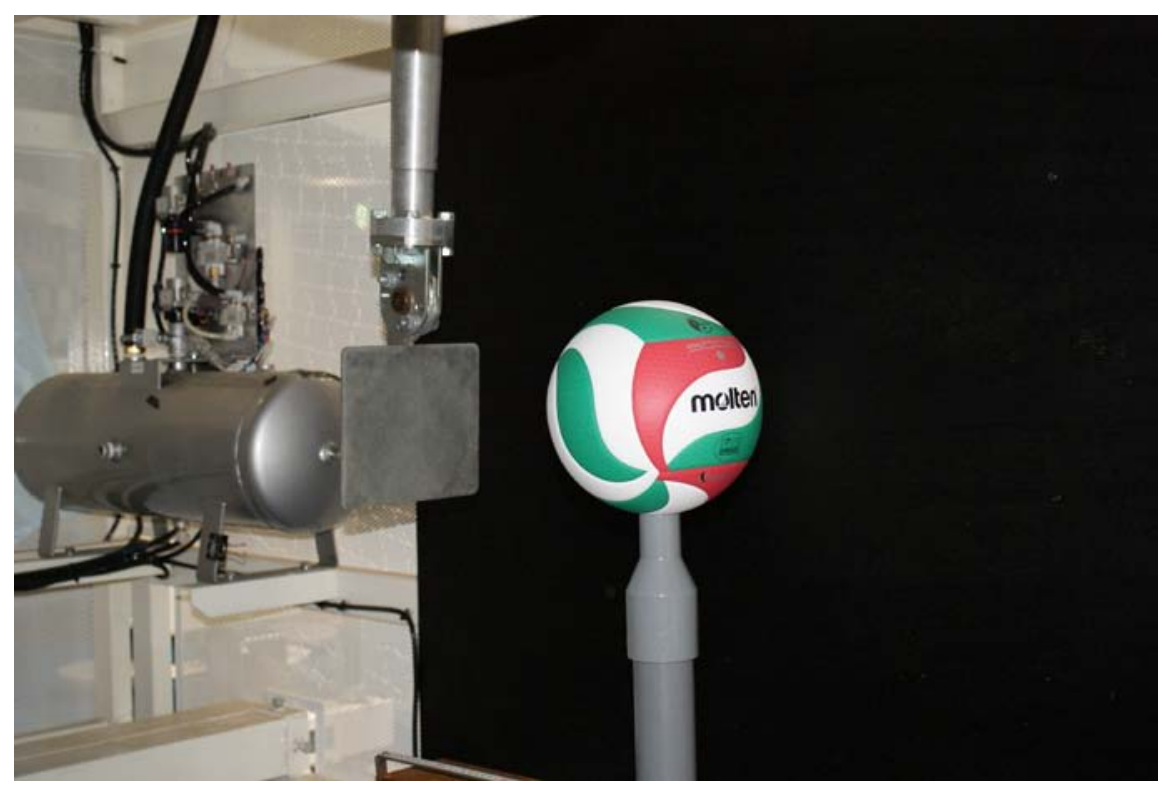

Figure 3. Set up of the impact-type ball ejection device (b). 

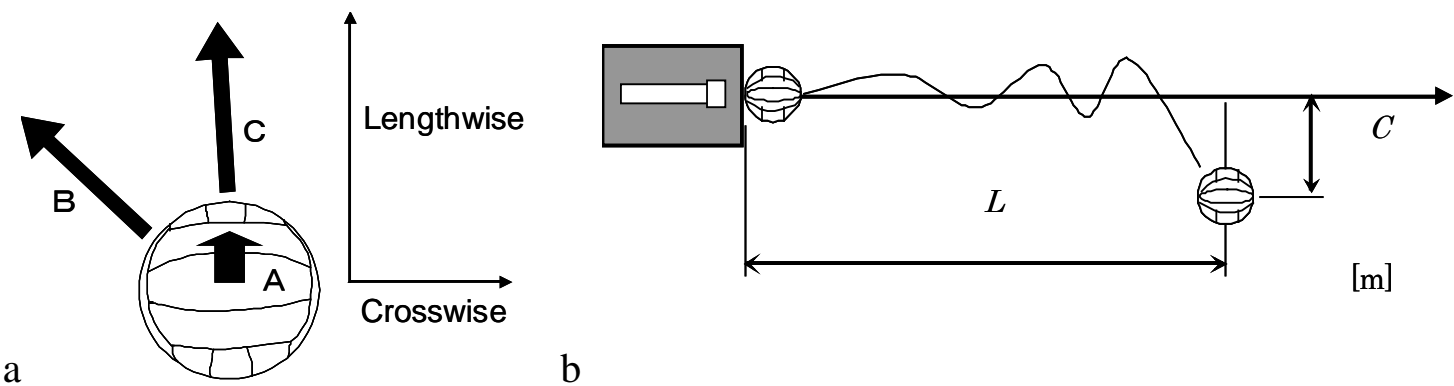

b

Figure 4. Orientation of ball panels on volley ball (a) and Set up for the aerodynamic instability test (b). 


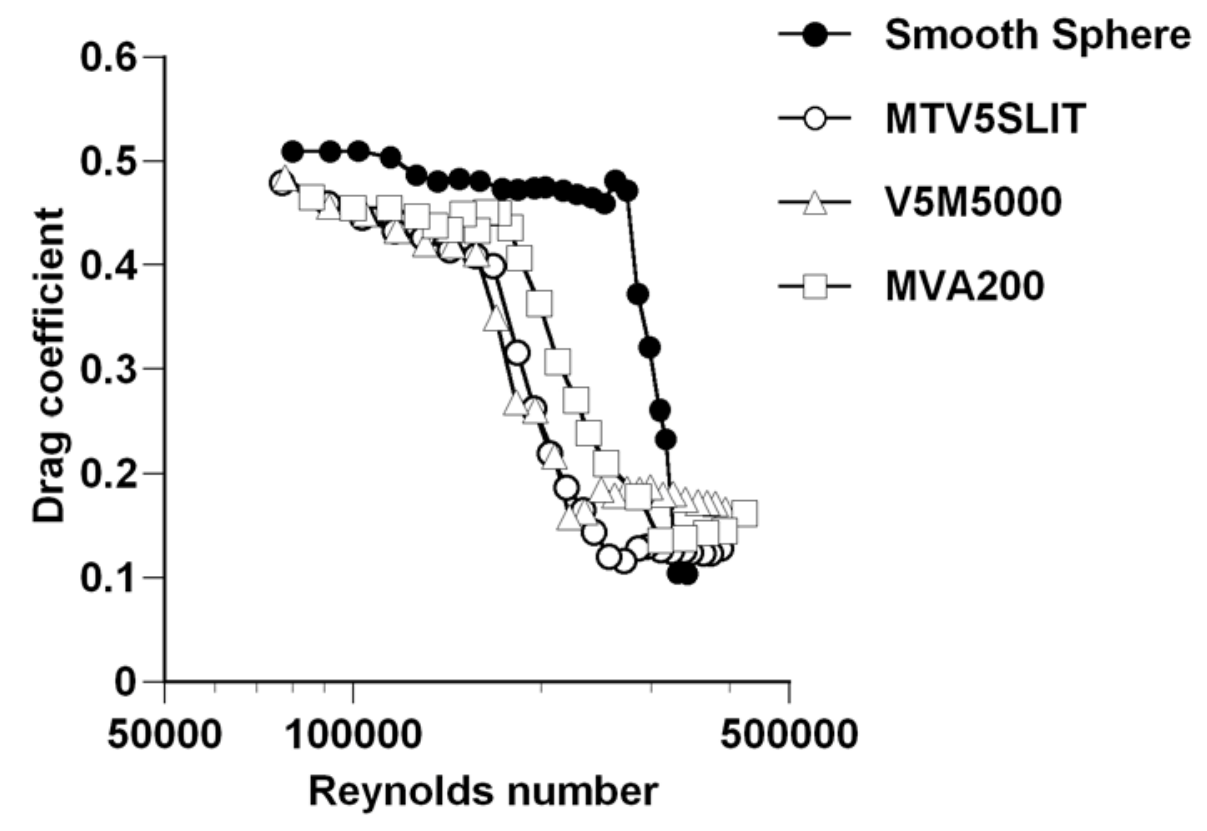

Figure 5. Drag coefficient versus Reynolds number for conventional type (Old) and new type (New) volleyballs. 


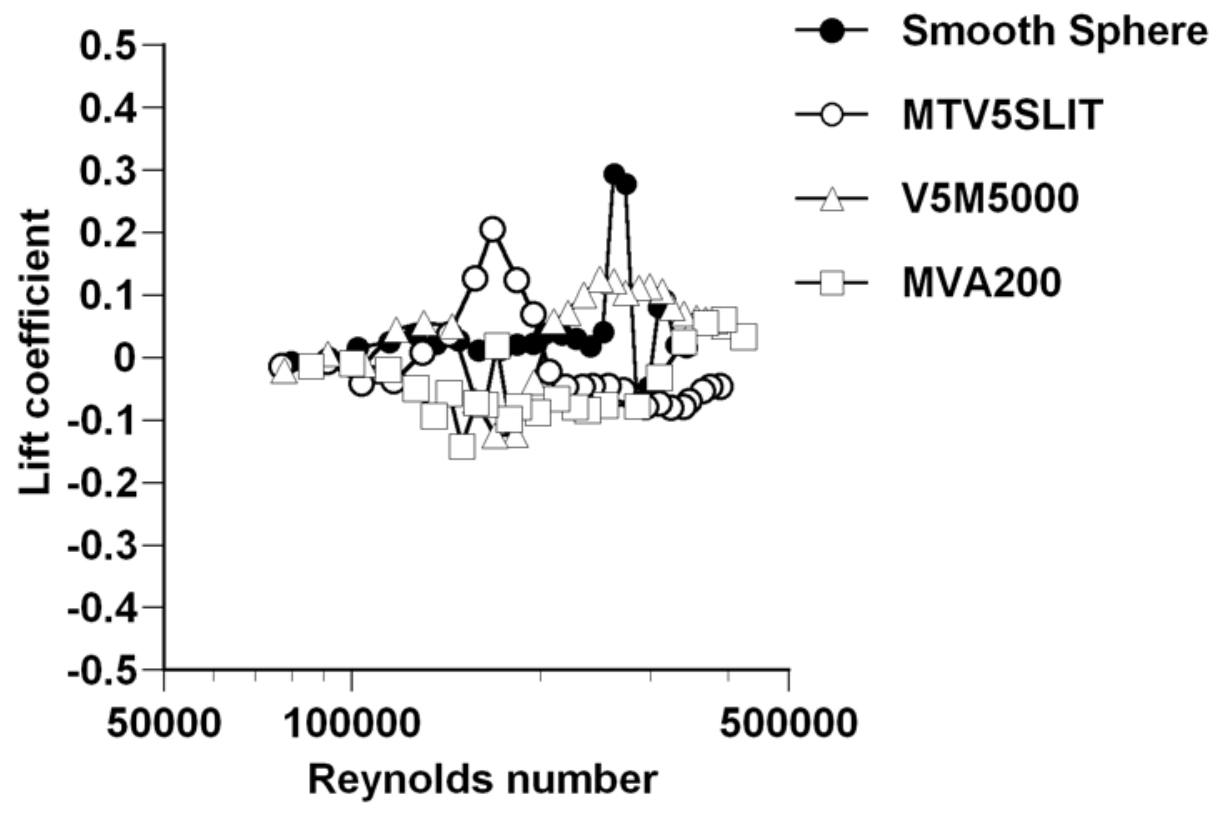

Figure 6. Lift coefficient versus Reynolds number for conventional type (Old) and new type (New) volleyballs. 

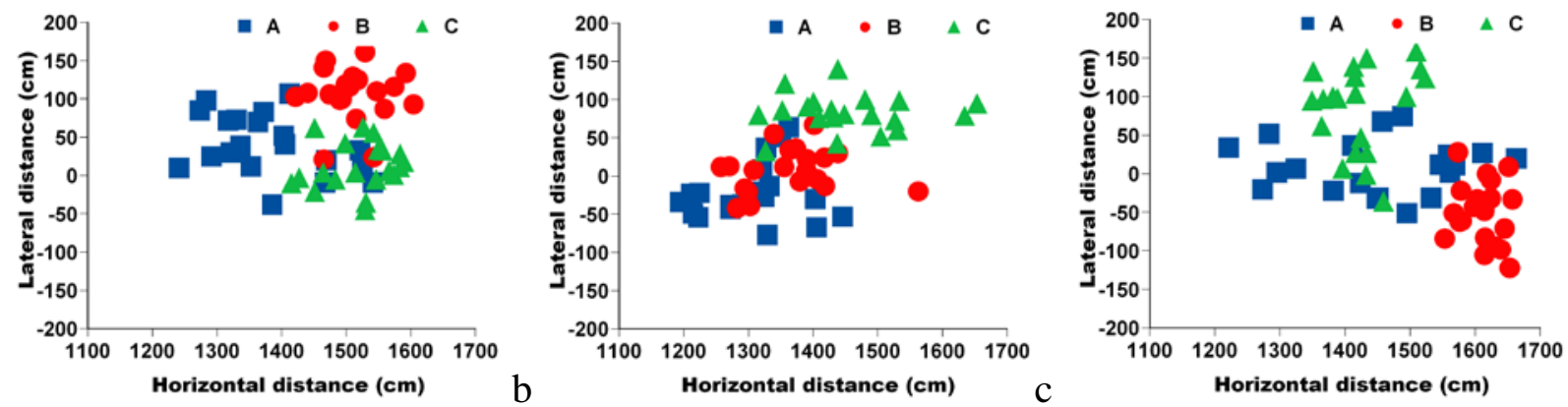

Figure 7. Impact (landing) points for honeycomb type ball (a), conventional type ball (b) and dimple type ball (c) ejected from the impact type ball ejection device (A, B, and C in the figure show the orientation of the ball panels). 


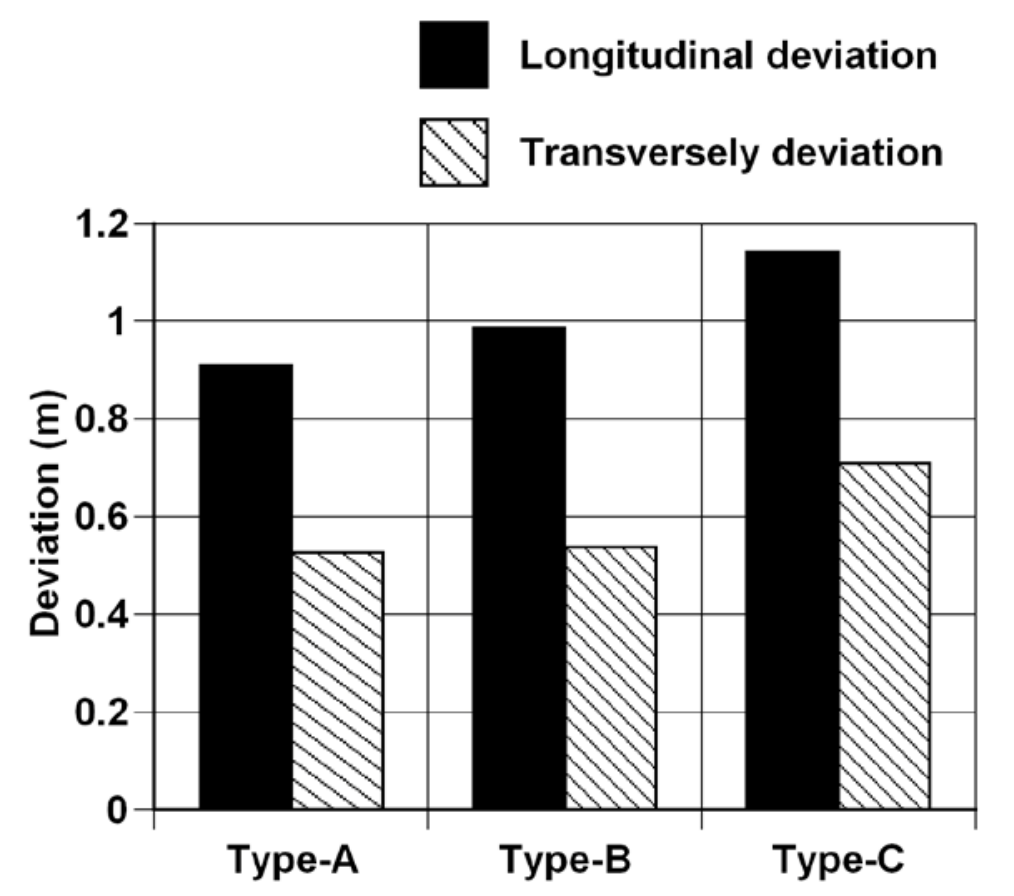

Figure 8. Standard deviations of longitudinal and transversal displacement for honeycomb type ball (Type-A), conventional type ball (Type-B) and dimple type ball (Type-C). 